PROCEEDINGS OF THE

AMERICAN MATHEMATICAL SOCIETY

Volume 125, Number 2, February 1997, Pages 339-345

S 0002-9939(97)03673-3

\title{
AN ENGEL CONDITION WITH DERIVATION FOR LEFT IDEALS
}

\author{
CHARLES LANSKI
}

(Communicated by Ken Goodearl)

\begin{abstract}
We generalize a number of results in the literature by proving the following theorem: Let $R$ be a semiprime ring, $D$ a nonzero derivation of $R, L$ a nonzero left ideal of $R$, and let $[x, y]=x y-y x$. If for some positive integers $t_{0}, t_{1}, \ldots, t_{n}$, and all $x \in L$, the identity $\left[\left[\ldots\left[\left[D\left(x^{t_{0}}\right), x^{t_{1}}\right], x^{t_{2}}\right], \ldots\right], x^{t_{n}}\right]=0$ holds, then either $D(L)=0$ or else the ideal of $R$ generated by $D(L)$ and $D(R) L$ is in the center of $R$. In particular, when $R$ is a prime ring, $R$ is commutative.
\end{abstract}

In this paper we prove a theorem generalizing several results, principally [20] and [9], which combine derivations with Engel type conditions. Before stating our theorem we discuss the relevant literature. If one defines $[x, y]_{0}=x$ and $[x, y]_{1}=$ $[x, y]=x y-y x$, then an Engel condition is a polynomial $[x, y]_{n+1}=\left[[x, y]_{n}, y\right]$ in noncommuting indeterminates. A commutative ring satisfies any such polynomial, and a nilpotent ring satisfies one if $n$ is sufficiently large. The question of whether a ring is commutative, or nilpotent, if it satisfies an Engel condition goes back to the well known work of Engel on Lie algebras [15, Chapter 2], and has been considered, with various modifications, by many since then (e.g. [2] or [7]). The connection of Engel type conditions and derivations appeared in a well known paper of E. C. Posner [23] which showed that for a nonzero derivation $D$ of a prime ring $R$, if $[D(x), x]$ is central for all $x \in R$, then $R$ is commutative. This result has led to many others (see [19] for various references), and in particular to a result of J. Vukman [25] showing that if $[D(x), x]_{2}$ is central for all $x \in R$, a prime ring with char $R \neq 2,3$, then again $R$ is commutative. We extended this result [20] by proving that if $[D(x), x]_{n}=0$ for all $x \in I$, an ideal of the prime ring $R$, then $R$ is commutative, and if instead, this Engel type condition holds for all $x \in U$, a Lie ideal of $R$, then $R$ embeds in $M_{2}(F)$ for $F$ a field with char $F=2$. Recently, [9] proved that for a left ideal $L$ of a semiprime ring $R$, either $D(L)=0$ or $R$ contains a nonzero central ideal if either: $R$ is 6 -torsion free and $[D(x), x]_{2}$ is central for all $x \in L$; or if $\left[D(x), x^{n}\right]$ is central for all $x \in L$ and $R$ is $n$ !-torsion free. The first of these conditions generalized [1, Theorem 3, p. 99], which assumed that $[D(x), x]$ is central for all $x \in L$, with no restriction on torsion. The second, involving powers, is related to both [12], which showed that a prime ring $R$ is commutative if $D\left(x^{k}\right)=0$ for all $x \in R$, and to [8], a significant extension of [12], showing that $R$ is commutative if it contains no nonzero nil ideal and $\left[D\left(x^{k(x)}\right), x^{k(x)}\right]_{n}=0$ on

Received by the editors August 2, 1995.

1991 Mathematics Subject Classification. Primary 16W25; Secondary 16N60, 16 U80.

(c)1997 American Mathematical Society 
$R$. Other results and conditions involving the image of a derivation on a one-sided ideal of $R$ have been appearing with increased frequency (e.g. [3], [4], [21], [24]).

Our result here combines a variant of the Engel condition and the action of a derivation on a left ideal in a semiprime ring. It generalizes or extends a number of the results mentioned above and eliminates all torsion assumptions.

Main Theorem. Let $R$ be a semiprime ring, $D$ a nonzero derivation of $R$, and $L$ a nonzero left ideal of $R$. If for some positive integers $t_{0}, t_{1}, \ldots, t_{n}$, and all $x \in L$, the identity $\left[\left[\ldots\left[\left[D\left(x^{t_{0}}\right), x^{t_{1}}\right], x^{t_{2}}\right], \ldots\right], x^{t_{n}}\right]=0$ holds, then either $D(L)=0$ or else $D(L)$ and $D(R) L$ are contained in a nonzero central ideal of $R$. In particular, when $R$ is a prime ring, $R$ is commutative.

Note that the statement about prime rings does follow from the semiprime case since if $I$ is a central ideal in a prime ring $R$, then the identity $[x y, z]=x[y, z]+$ $[x, z] y$ shows that $0=[I R, R]=I[R, R]$, so $I=0$ or $R$ is commutative. Also, when $R$ is prime and $D(L)=0$, then $D(R) L=D(R L)=0$, and $D=0$ results. Something like the conclusion that $R$ contains a central ideal is the most that one can expect since $R$ could be the direct sum of ideals $A, B$, and $G$, with $G$ commutative, $I=B+G, D(A) \neq 0, D(B)=0$ and $D(G) \subseteq G$. In this case $D(I)$ is central but $D(I) \neq 0$ and $I$ itself is not central.

The heart of our proof of the Main Theorem is a special case for prime rings. The basic approach and ideas are like those in [20], so we first recall the basic notions required ([6] or [18]). If $R$ is a prime ring, its extended centroid $C(R)=C$ is a field which is the center of the symmetric quotient ring $Q=Q(R)$ of $R$. For our purposes it suffices to know that $R C$ and $Q$ are prime overrings of $R$, for each $q \in Q$ there is a nonzero ideal $I_{q}$ of $R$ with $q I_{q}+I_{q} q \subseteq R$, and if $q I_{q}=0$, then $q=0$. Any derivation $D$ of $R$ extends uniquely to $Q$, and if on $Q, D(q)=q A-A q$ for $A \in Q$, then $D$ is called inner; otherwise $D$ is outer. An important result of W. S. Martindale [22] is that $R$ satisfies a generalized polynomial identity exactly when $H=\operatorname{soc} R C \neq 0$ and for each minimal left ideal $R C e$ of $R C$ with $e^{2}=e$, $e R C e$ is a finite dimensional divisional algebra over $C$.

Theorem 1. Let $R$ be a prime ring, $D$ a nonzero derivation of $R$, and $L$ a nonzero left ideal of $R$. If for integers $k, n+1 \geq 1,\left[D\left(x^{k}\right), x^{k}\right]_{n}=0$ for all $x \in L$, then $R$ is commutative.

Proof. It is easy to see that if $L \subseteq R \cap C$, then $R$ must be commutative [14, Corollary, p. 7], so we may choose $a \in L-C$. For any $r \in R,\left[D\left((r a)^{k}\right),(r a)^{k}\right]_{n}=0$, and it follows that

$$
\left[D\left((X a)^{k}\right),(X a)^{k}\right]_{n}=\left[\sum_{i=0}^{k-1}(X a)^{i}\left(X^{D} a+X D(a)\right)(X a)^{k-i-1},(X a)^{k}\right]_{n}
$$

is an identity with derivation which is satisfied by $R$. If $D$ is an outer derivation, a direct application of [17, Theorem 2, p. 65] or [6, Main Theorem, p. 251], together with $\left[5\right.$, Theorem 2, p. 725] show that $\left[\sum_{i=0}^{k-1}(X a)^{i}(Y a+X D(a))(X a)^{k-i-1},(X a)^{k}\right]_{n}$ is an identity for $Q$, which yields easily that $\left[\sum_{i=0}^{k-1}(X a)^{i}(Y a)(X a)^{k-i-1},(X a)^{k}\right]_{n}$ is an identity for $Q$ by first setting $Y=0$. Since $a \notin C$, this identity is a nonzero 
generalized polynomial identity for $R$, so by Martindale's theorem [22, Theorem 3 , p. 579] $H=\operatorname{soc} R C \neq 0$. Clearly the identity holds on $H \subseteq Q$. If $H$ is commutative, then so is $R$ and we are finished. Otherwise, since $H a \subseteq H$ [18, Lemma 7, p. 779], there is a minimal left ideal $H e \subseteq H a$ with $e^{2}=e \in H$ and $H t a=H e$ for some $t \in H$. Consequently, $H e$ satisfies $\left[\sum_{i=0}^{k-1} X^{i} Y X^{k-i-1}, X^{k}\right]_{n}=0$. Evaluating this expression with $X=h e$ and $Y=(1-e) y e$ for arbitrary $h, y \in H$, and using $h e(1-e) y e=0$ results in $(1-e) y e(h e)^{k(n+1)-1}=0$. Because $H e$ is minimal, if $(1-e) y e \neq 0$, it follows that $H e=H(1-e) y e$, so $(h e)^{k(n+1)}=0$ results. This means that $\mathrm{He}$ is a nil left ideal of bounded index and Levitzki's theorem [13, Lemma 1.1, p. 1] forces $R$ to contain a nonzero nilpotent ideal. This contradiction shows that $R$ must be commutative when $D$ is outer.

We may now take $D(q)=[q, A]$ with $A \in Q-C$, since $D \neq 0$. As above, if we choose $a \in L-C$, then our assumption yields the identity $\left[A,(r a)^{k}\right]_{n+1}=0$ for $R$. This is a nonzero generalized polynomial identity because $A \notin C$, so Martindale's theorem [22, Theorem 3, p. 579] shows that $H=\operatorname{soc} R C \neq 0$ and $e H e$ is finite dimensional over $C$ for $e^{2}=e$ a minimal idempotent in $H$. Now the identity $\left[A,(X a)^{k}\right]_{n+1}$ is also satisfied by $Q[5$, Theorem 2, p. 725] and hence by $H$. As in the case above, $R$ is commutative if $H$ is, so we proceed with the assumption that $H$ is not commutative to get the contradiction $D=0$.

We want to replace $R$ with $H$ and be able to assume that for any minimal idempotent $e \in H, C e=e H e$. We note that $C=C(H), C H=H$ and $D(H) \subseteq H$ [18, Lemma 7, p. 59], and $C$ centralizes $H$, so it is clear that $C e \subseteq Z(e H e)$ for any idempotent $e \in H$. Assume first that $C$ is a finite field. From the finite dimensionality of $e \mathrm{He}$ over $\mathrm{Ce}$ it follows that $\mathrm{eHe}$ is a finite field, so for $z \in e \mathrm{He}$ and any $h \in H$, zehe $=e h e z$, which forces $z e=c e$ for $c \in C(H)=C[22$, Theorem 1, p. 577]. Therefore $C e=Z(e H e)=e H e$ when $C$ is a finite field. If $C$ is infinite, then a Vandermonde determinant argument, for example that in [20, Lemma 2, p. 732], shows that $\left[A,(X a)^{k}\right]_{n+1}$ is satisfied by any extension $H \otimes_{C} F$ of $H$, for $F$ a field extension of $C$. In particular we can take $F$ to be an algebraic closure of $C$. Now $C\left(H \otimes_{C} F\right)=F\left[10\right.$, Theorem 3.5, p. 59], $\operatorname{soc}\left(R C \otimes_{C} F\right)=H \otimes_{C} F$, and for any minimal idempotent $e \in H \otimes_{C} F, e\left(H \otimes_{C} F\right) e$ is finite dimensional over $e F$, again by [22], so $e\left(H \otimes_{C} F\right) e=e F$ because $F$ is algebraically closed. Consequently, regardless of card $C$, we may assume that $H=R$ and $e C=e H e$ for any minimal idempotent $e \in H$.

Since $H$ satisfies the identity $\left[A,(X a)^{k}\right]_{n+1}$, as for the case above when $D$ was assumed to be outer, for some minimal idempotent $e \in H$ and some $t \in H, H e=$ $H t a$ satisfies the identity $\left[A, X^{k}\right]_{n+1}$. In particular if $X=e$ we obtain $[A, e]_{n+1}=0$ and also $[A, e]_{n+2}=0$. Since one of $n+1$ or $n+2$ is odd and $[A, e]=[A, e]_{3}$, it follows immediately that $[A, e]=0$, and we may write $A=e A e+(1-e) A(1-e)$. But $e A e=e(A e) e \in e H e=C e$, so $A=c e+(1-e) A(1-e)$. For any $h \in H$ we evaluate $\left[A,(h e)^{k}\right]_{n+1}=0$ using the identities $[y, x]_{n+1}=\sum_{i=0}^{n+1}(-1)^{i}\left(\begin{array}{c}n+1 \\ i\end{array}\right) x^{i} y x^{n+1-i}$ and $[x+y, z]_{s}=[x, z]_{s}+[y, z]_{s}$ to obtain

$$
\begin{aligned}
0= & (1-e) A(1-e)(h e)^{k(n+1)}+\sum_{i=0}^{n+1}(-1)^{i}\left(\begin{array}{c}
n+1 \\
i
\end{array}\right)(h e)^{k i} e c(h e)^{k(n+1-i)} \\
= & (1-e) A(1-e)(h e)^{k(n+1)}+e c(h e)^{k(n+1)} \\
& +c \sum_{i=1}^{n+1}(-1)^{i}\left(\begin{array}{c}
n+1 \\
i
\end{array}\right)(h e)^{k i} e(h e)^{k(n+1-i)}
\end{aligned}
$$




$$
\begin{aligned}
& =(1-e) A(1-e)(h e)^{k(n+1)}+e c(h e)^{k(n+1)}-c(h e)^{k(n+1)} \\
& =(1-e) A(1-e)(h e)^{k(n+1)}-(1-e) c(h e)^{k(n+1)} \\
& =(1-e)(A-c)(1-e)(h e)^{k(n+1)} .
\end{aligned}
$$

Hence $(A-c)(h e)^{k(n+1)}=(A-c)(e+(1-e))(h e)^{k(n+1)}=(A-c)(1-e)(h e)^{k(n+1)}=0$ since $A e=c e$ and $(A-c)(1-e)=(1-e)(A-c)(1-e)$. A result of B. Felzenszwalb [11, Theorem 2, p. 242] shows that in a ring with no nonzero nil left ideal, if $y t^{s}=0$ for all $t \in L$, a nonzero left ideal, then $y L=0$. Therefore, we have $(A-c) H e=0$, forcing $A=c \in C$ and $D=0$, a contradiction. Consequently, $R$ must be commutative, completing the proof of the theorem.

The special case of Theorem 1 when $n=0$ gives [12, Theorem 2, p. 19], since $k D(x) x^{k-1}=D\left(x^{k}\right)=0$ forces char $R=p \mid k$. Also, the theorem is a version of [8, Corollary 1, p. 36] for left ideals where we must assume that the exponents $k$ are fixed but need not assume that $R$ has no nil ideal. Before proving our Main Theorem, it will be helpful to collect a few observations together into a lemma.

Lemma. Let $R$ be a semiprime ring and $M$ the maximal central ideal of $R$.

(1) $M=\operatorname{ann}([R, R])$ is a semiprime ideal of $R$;

(2) if $a \in R$ and $R a$ is central, then $a \in M$; and

(3) if $D$ is a derivation of $R$, then $D(M) \subseteq M$.

Proof. Since any annihilator ideal in a semiprime ring is a semiprime ideal, it suffices to show that $M=\operatorname{ann}([R, R])$ to prove (1). Let $A=\operatorname{ann}([R, R])$ and note that $0=[M R, R]=M[R, R]$, so $M \subseteq A$. But $[A, R] \subseteq A \cap([R, R])=0$ since $R$ is semiprime, and $A=M$. Next observe that $R / M$ has no nonzero central ideal. If $M \subseteq I$ is an ideal of $R$ with $I / M$ central in $R / M$, then $[I, R] \subseteq M$ implies that $[[I, R], R]=0$, so $[I,[R, R]]=0$ and $I$ is central by $[14$, Lemma 1.1 .8$, p. 8$]$ forcing $I=M$. Consequently, if $R a+M$ is central in $R / M$, then $R a \subseteq M$, which results in $a \in M$ by (1). Finally, for any derivation $D$ it is easy to see that $D(Z(R)) \subseteq Z(R)$, the center of $R$, and then that $M+D(M)$ is an ideal of $R$ in $Z(R)$. Thus $D(M) \subseteq M$ by the maximality of $M$.

Proof of Main Theorem. Our assumption that $\left[\left[\ldots\left[\left[D\left(x^{t_{0}}\right), x^{t_{1}}\right], x^{t_{2}}\right], \ldots\right], x^{t_{n}}\right]=0$ for all $x \in L$ implies that $\left[D\left(x^{k}\right), x^{k}\right]_{n}=0$ for $k=t_{0} t_{1} \cdots t_{n}$ since powers of $x$ commute, so we may as well assume that all $t_{j}=k$. We claim that $R D(R) L$ is a central ideal of $R$, and is not zero unless $D(L)=0$. Should $D(R) L=0$, then $L \subseteq \operatorname{ann}(D(R))$, the left or right annihilator of $(D(R))$, the ideal $D(R)$ generates. It is easy to see that $D(L) \subseteq D(\operatorname{ann}(D(R))) \subseteq D(R) \cap \operatorname{ann}(D(R))=0$, since $R$ is semiprime. Consequently, to prove the existence of a nonzero central ideal, it suffices to assume that $D(L) \neq 0$ and show that $R D(R) L$ is central. Equivalently, we need to prove that for each prime ideal $P$ of $R$, the image of $R D(R) L$ is central in $R / P$. This is clear if $D(R) L \subseteq P$, so we need only consider those prime ideals with $D(R) L \not \subset P$.

Let $P$ be a prime ideal of $R$ so that $D(R) L \not \subset P$, and suppose that $D(P) \subseteq P$. In this case, $D$ induces a derivation $E$ on $R / P$ via $E(r+P)=E(r)+P$ and our hypothesis carries over from $R$ to $R / P$ using $E$ and the left ideal $L+P \subseteq R / P$. Applying Theorem 1 gives either $E=0, L+P \subseteq P$, or $R / P$ commutative. Since the first two possibilities each force $D(R) L \subseteq P$, we must conclude that $R / P$ is commutative, so $R D(R) L+P$ is central in $R / P$. 
We may assume now that $D(R) L \not \subset P$ and $D(P) \not \subset P$. It is straightforward to check that $D(P)+P=B \subseteq R / P$ is a nonzero ideal. For any $t \in P$ and $y \in$ $L$ our assumption that $\left[D\left((t y+y)^{k}\right),(t y+y)^{k}\right]_{n}=0$, taken modulo $P$ becomes $\left[\sum_{i=0}^{k-1} y^{i}(D(t) y+D(y)) y^{k-i-1}, y^{k}\right]_{n}=0$ in $R / P$. But

$$
\left[\sum_{i=0}^{k-1} y^{i} D(y) y^{k-i-1}, y^{k}\right]_{n}=\left[D\left(y^{k}\right), y^{k}\right]_{n}=0,
$$

so $\left[\sum_{i=0}^{k-1} y^{i} D(t) y^{k-i}, y^{k}\right]_{n}=0$ in $R / P$, which means that the expression $f(X, Y)=$ $\left[\sum_{i=0}^{k-1} Y^{i} X Y^{k-i}, Y^{k}\right]_{n}$ yields $O_{R / P}$ when elements of $B$ replace $X$ and elements of $L+P$ replace $Y$. If for some $y \in(L+P)-P$, yw $=0$ in $R / P$ for $w \in$ $R / P-O_{R / P}$, then for any $b \in B$ and $r \in R, O_{R / P}=f(w b, r y)=w b(r y)^{k(n+1)}$. Thus $w B(r y)^{k(n+1)}=0$ in $R / P$, and since $B$ is a nonzero ideal and $R / P$ is prime, we must conclude that $R y+P$ is a nil left ideal of bounded index in $R / P$, forcing the contradiction $y \in P$ by Levitzki's theorem [13, Lemma 1.1, p. 1]. Therefore, we may assume that each nonzero $y \in L+P$ has no right annihilator in $R / P$.

To simplify notation, we assume that $R$ is a prime ring with a nonzero ideal $B$ and nonzero left ideal $L$ whose nonzero elements are left regular, that $f(X, y)$ is an identity for $B$ for each $y \in L$, and show that $R$ is commutative. Expanding $f(X, y)$ for $y \in L-0$, yields the identity $\sum_{j=0}^{v} n_{j} y^{j} X y^{v-j}$ for $B$, where $n_{j}$ are integers, $n_{0}=1$, and $v=k(n+1)$. This is a generalized linear identity for $B$, so by $[18$, Lemma 1 , p. 766$],\left\{1, y, \ldots, y^{v}\right\}$ must be $C(R)$ dependent. Let $m(y)=y^{s}+\cdots+c_{1} y+c_{0}=0$ with $c_{i} \in C(R)$ and $s$ minimal. The definition of $Q$ allows us to choose a nonzero ideal $I$ of $R$ so that all $c_{i} I \subseteq R$. Thus if $c_{0}=0$ and $m(y)=y g(y)$, then $g(y) I \subseteq R$, so $g(y) I$ is in the right annihilator of $y$, and $g(y) I=0$ forces $g(y)=0$, contradicting the minimality of $s$. Therefore $c_{0} \neq 0$ and $J=c_{0} I=I c_{0} \subseteq R y$. Now $f(X, Y)$ is a polynomial identity for $B \cap J \subseteq L$, and so for its central localization, a finite dimensional central simple algebra [16, Theorem 2, p. 57]. Applying [20, Lemma 2, p. 732] shows that $B \cap J$ is commutative or that $f(X, Y)$ is an identity for some $M_{d}(F)$ for $F$ a field and $d>1$. But $f\left(e_{12}, e_{22}\right)=e_{12} \neq 0$, for $e_{12}$ and $e_{22}$ matrix units in $M_{d}(F)$, so $B \cap J$ is commutative, forcing $R$ to be commutative [14, Corollary, p. 7], and showing that our original semiprime ring must contain the nonzero central ideal $R D(R) L$.

Finally, we must show that $D(L), D(R) L \subseteq M$, the maximal central ideal of our semiprime ring $R$. We have just proven that $R D(R) L \subseteq M$, so by the Lemma $D(R) L \subseteq M$ and $D(R) D(L) \subseteq D(D(R) L)+D^{2}(R) L \subseteq D(M)+M=M$. Hence

$$
D(L) R D(L) \subseteq D(L R) D(L)+M \subseteq M,
$$

and the semiprimeness of $M$ by the Lemma forces $D(L) \subseteq M$. Therefore, the proof of the Main Theorem is complete.

It is clear that the Main Theorem generalizes both [9] and [20], and in the way we mentioned after Theorem 1, [8] as well. We end the paper with another consequence of the Main Theorem by giving an extension to one-sided ideals of [2, Theorem 3, p. 385] and [7, Theorem 2, p. 120].

Theorem 2. Let $R$ be a semiprime ring and $L$ a nonzero left ideal of $R$. If for integers $n, k \geq 1$, and some $a \in R,\left[a, x^{k}\right]_{n}=0$ for all $x \in L$, then $[a, L]=0$. When $R$ is a prime ring, then $a \in Z(R)$, the center of $R$. 
Proof. Define a derivation $D$ of $R$ by $D(r)=[r, a]$. Then for all $x \in L$,

$$
-\left[D\left(x^{k}\right), x^{k}\right]_{n-1}=\left[-D\left(x^{k}\right), x^{k}\right]_{n-1}=\left[a, x^{k}\right]_{n}=0 .
$$

By the Main Theorem, either $D=0$ or $D(L) \subseteq Z(R)$. When $D=0, a \in Z(R)$ is immediate, and when $D(L) \subseteq Z(R)$, [[a,L],R] $=0$. In particular, if $y \in L$ and $r \in R$, then

$$
0=[[a, a y], r]=[a[a, y], r]=[a, r][a, y],
$$

so letting $r=y s$ for $s \in R$ shows that $[a, y] R[a, y]=0$. Since $R$ is semiprime we are forced to conclude that $[a, L]=0$. When $R$ is prime, $0=[a, R L]=[a, R] L$, so $a \in Z(R)$, proving the theorem.

\section{REFERENCES}

1. H. E. Bell and W. S. Martindale, III, Centralizing mappings of semiprime rings, Canad. Math. Bull. 30 (1987), 92-101. MR 88h:16044

2. H. E. Bell and I. Nada, On some center-like subsets of rings, Arch. Math. 48 (1987), 381-387. MR 88h: 16045

3. M. Brešar, Centralizing mappings and derivations in prime rings, J. Algebra 156 (1993), 385-394. MR 94f: 16042

4. M. Brešar, One-sided ideals and derivations of prime rings, Proc. Amer. Math. Soc. 122 (1994), 979-983. MR 95b:16037

5. C. L. Chuang, GPIs having coefficients in Utumi quotient rings, Proc. Amer. Math. Soc. 103 (1988), 723-728. MR 89e:16028

6. C. L. Chuang, ${ }^{*}$-differential identities of prime rings with involution, Trans. Amer. Math. Soc. 316 (1989), 251-279. MR 90b:16018

7. C. L. Chuang and J. S. Lin, On a conjecture by Herstein, J. Algebra 126 (1989), 119-138. MR 90i: 16028

8. C. L. Chuang, Hypercentral derivations, J. Algebra 66 (1994), 34-71. MR 95e:16029

9. Q. Deng and H. E. Bell, On derivations and commutativity in semiprime rings, Comm. Algebra 23 (1995), 3705-3713. CMP 95:17

10. T. S. Erickson, W. S. Martindale, III, and J. M. Osborn, Prime nonassociative algebras, Pacific J. Math. 60 (1975), 49-63. MR 52:3264

11. B. Felzenszwalb, On a result of Levitzki, Canad. Math. Bull. 21 (1978), 241-242. MR 58:10992

12. B. Felzenszwalb, Derivations in prime rings, Proc. Amer. Math. Soc. 84 (1982), 16-20. MR 83b: 16030

13. I. N. Herstein, Topics in ring theory, University of Chicago Press, Chicago, 1969. MR 42:6018

14. I. N. Herstein, Rings with involution, University of Chicago Press, Chicago, 1976. MR 56:406

15. N. Jacobson, Lie algebras, Wiley, New York, 1962; reprint, Dover, New York, 1979. MR 26:1345; MR 80k:17001

16. N. Jacobson, PI-algebras, Lecture Notes in Math., Vol. 441, Springer-Verlag, New York, 1975. MR 51:5654

17. V. K. Kharchenko, Differential identities of semiprime rings, Algebra and Logic 18 (1979), 58-80. MR 81f:16052 (of Russian original)

18. C. Lanski, Differential identities in prime rings with involution, Trans. Amer. Math. Soc. 291 (1985), 765-787. MR 87f:16013

19. C. Lanski, Differential identities, Lie ideals, and Posner's theorems, Pacific J. Math. 134 (1988), 275-297. MR 89j:16051

20. C. Lanski, An Engel condition with derivation, Proc. Amer. Math. Soc. 118 (1993), 731-734. MR 93i: 16050

21. C. Lanski, Derivations with nilpotent values on left ideals, Comm. Algebra 22 (1994), 13051320. MR 95h:16048

22. W. S. Martindale, III, Prime rings satisfying a generalized polynomial identity, J. Algebra 12 (1969), 576-584. MR 39:257

23. E. C. Posner, Derivations in prime rings, Proc. Amer. Math. Soc., 8 (1957), 1093-1100. MR 20:2361 
24. B. Tilly, Derivations whose iterates are zero or invertible on a left ideal, Canad. Math. Bull. 37 (1994), 124-132. MR 94m:16041

25. J. Vukman, Commuting and centralizing mappings in prime rings, Proc. Amer. Math. Soc. 109 (1990), 47-52. MR 90h:16010

Department of Mathematics, University of Southern California, Los Angeles, CalIFORNIA 90089-1113

E-mail address: clanski@math.usc.edu 